\title{
Drone Sembrador
}

\section{Sower Drone}

\author{
TENORIO-CRUZ, Fermín†**, JUÁREZ-CORTÉS, Erik, PÉREZ-VILLEGAS, Manuel y TÉLLEZ- \\ HERNÁNDEZ, Rubén
}

Universidad Tecnológica de Tecamachalco

Benemérita Universidad Autónoma de Puebla

ID $1^{\text {er }}$ Autor: Fermín, Tenorio-Cruz / ORC ID: 0000-0002-8727-1502, Researcher ID Thomson: S-8629-2018, CVU CONACYT ID: 84030

ID $1^{\text {er }}$ Coautor: Erik, Juárez-Cortés / ORC ID: 0000-0002-4478-0825, Researcher ID Thomson: S-8738-2018, CVU CONACYT ID: 947648

ID $2^{\text {do }}$ Coautor: Manuel, Pérez-Villegas / ORC ID: 0000-0002-4204-3813, CVU CONACYT ID: 818732

ID $3^{\text {er }}$ Coautor: Ruben, Téllez-Hernández / ORC ID: 0000-0002-6184-3226, Researcher ID Thomson: S-8747-2018, CVU CONACYT ID: 665940

DOI: $10.35429 / J T I P .2020 .11 .4 .8 .12$

Recibido 03 de Agosto, 2020; Aceptado 20 Diciembre, 2020

\section{Resumen}

Utilizar tecnología en la agricultura debe ayudar a incrementar la producción y disminuir los riesgos que sufre el agricultor al realizar los diferentes procesos como la siembra de muchos cultivos que se hace de manera manual dispersando la semilla con la mano. Al realizar el proceso de siembra de algún cultivo mediante la dispersión de su semilla con la mano, el agricultor desperdicia tiempo, puede sufrir lesiones e incluso fracturas al recorrer el terreno a sembrar y también, el agricultor al estar mucho tiempo expuesto a la radiación solar, tiene el riesgo de contraer cáncer en la piel. Nuestro proyecto busca resolver los tres puntos mencionados anteriormente y contribuir mediante la utilización de un dispositivo aéreo no tripulado y la modelación matemática de las trayectorias a seguir por parte del dispositivo, para realizar la distribución adecuada de la semilla sobre la superficie a sembrar y así el agricultor aumente su producción y disminuya los factores que le generan pérdidas. Pruebas realizadas indican que nuestro dron es completamente funcional y cumple con los objetivos planteados en el proyecto, contribuyendo a proporcionar gran ayuda en el desarrollo de la actividad del agricultor en la siembra por dispersión de semilla de cualquier cultivo.

Dron, Sembrador, Modelado

\begin{abstract}
Using technology in agriculture should help increase production and reduce the risks suffered by the farmer when carrying out the different processes such as the planting of many crops that is done manually by dispersing the seed by hand. When carrying out the process of sowing a crop by dispersing its seed by hand, the farmer wastes time, can suffer injuries and even fractures when traveling the field to be sown and also, the farmer being exposed to solar radiation for a long time, you are at risk for skin cancer. Our project seeks to solve the three points mentioned above and contribute through the use of an unmanned aerial device and the mathematical modeling of the trajectories to be followed by the device, to carry out the adequate distribution of the seed on the surface to be sown and thus the farmer increase his production and reduce the factors that generate losses. Tests carried out indicate that our drone is fully functional and meets the objectives set out in the project, helping to provide great help in the development of the farmer's activity in sowing by seed dispersal of any crop.
\end{abstract}

Drone, Sower, Modelling

Citación: TENORIO-CRUZ, Fermín, JUÁREZ-CORTÉS, Erik, PÉREZ-VILLEGAS, Manuel y TÉLLEZ-HERNÁNDEZ, Rubén. Drone Sembrador. Revista de Tecnologías en Procesos Industriales. 2020. 4-11: 8-12

\footnotetext{
* Correspondencia del Autor (fermin.tenorio@ @orreo.buap.mx)

$\dagger$ Investigador contribuyendo como primer autor.
} 


\section{Introducción}

En el mundo hay muchos cultivos cuya siembra comienza con el depósito de su semilla sobre la tierra. Este proceso todavía en la mayoría de los casos se viene realizando de forma manual. El agricultor con su mano va dispersando la semilla conforme recorre todo el terreno a sembrar.

El proceso anterior genera pérdida de tiempo para el agricultor, posibles fracturas o torceduras durante su recorrido para dispersar la semilla y estar expuesto mucho a la radiación solar con las posibles consecuencias que esto conlleva.

Este proyecto busca contribuir a la automatización del proceso de siembra de cultivos mediante la dispersión de sus semillas utilizando un dron para ello y su modelado matemático del dispositivo y las trayectorias a seguir para cumplir con tal función. Ya que la alfalfa actualmente es el cultivo forrajero más importante en el Mundo, en este proyecto se utilizó la semilla de alfalfa como la semilla para realizar las pruebas requeridas puesto que la alfalfa puede ser sembrada en cualquier época del año.

Además, México es de los principales productores mundiales de alfalfa y el estado de Puebla es considerado como uno de los principales estados de nuestro país productor de tal cultivo. De igual forma, el Municipio de Tecamachalco es de los principales municipios del estado de Puebla que producen alfalfa debido a que la agricultura es una de las principales actividades económicas de la región.

Estamos convencidos que cualquier área de conocimiento debe ser utilizada para resolver problemas reales y en particular, problemas del entorno de la región de origen de los investigadores en turno.

El proyecto está enfocado a resolver la situación en general de la siembra por dispersión de semilla de forma manual de cualquier cultivo mediante un dron, pero utilizamos solo semilla de alfalfa para realizar la validación de nuestra hipótesis puesto que para la siembra de otros cultivos mediante la dispersión de semilla sería similar.

\section{Objetivo del proyecto \\ General:}

- Establecer un modelo matemático para las trayectorias geométricas a seguir por un dron para realizar la siembra de semillas.

\section{Particular:}

- Automatizar el proceso de siembra con semillas para distintos cultivos.

\section{Descripción y funcionamiento}

La siembra manual de cualquier cultivo mediante la dispersión de semilla con la mano, se lleva a cabo de la siguiente forma: El agricultor tiene que cargar una bolsa con la semilla, caminar para abarcar todo el terreno a sembrar y dispersar con su mano la semilla contenida en la bolsa. La figura 1 muestra dicho proceso.

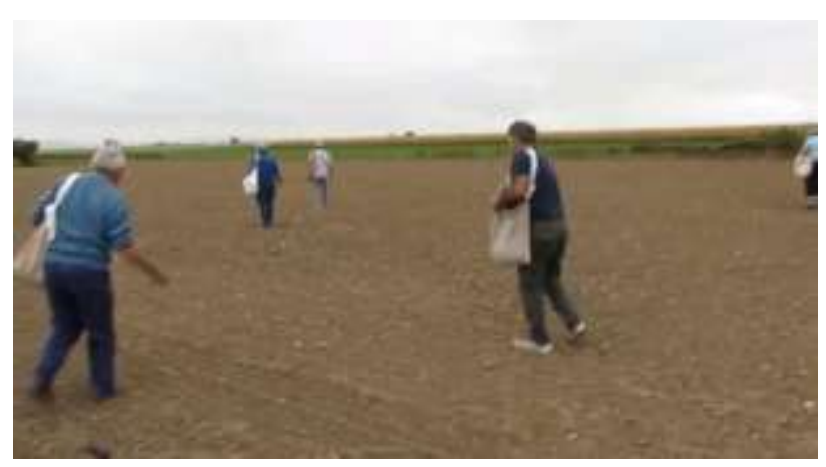

Figura 1 Siembra manual dispersando la semilla con la mano

La anterior forma de siembra da como resultado que, primero; el agricultor desperdicia tiempo, segundo, el agricultor puede sufrir lesiones e incluso fracturas al recorrer el terreno a sembrar y tercero, el agricultor al estar mucho tiempo expuesto a la radiación solar tiene el riesgo de contraer cáncer en la piel.

El presente proyecto consiste en evitar que el agricultor sufra los tres aspectos mencionados anteriormente. Para lograrlo, se realizó el diseño y construcción de un DRON al cual se le implementó un depósito que contenía la semilla de alfalfa a sembrar y se generó el modelo matemático para automatizar las trayectorias geométricas que seguiría.

En la siguiente figura se muestra el contenedor utilizado. 


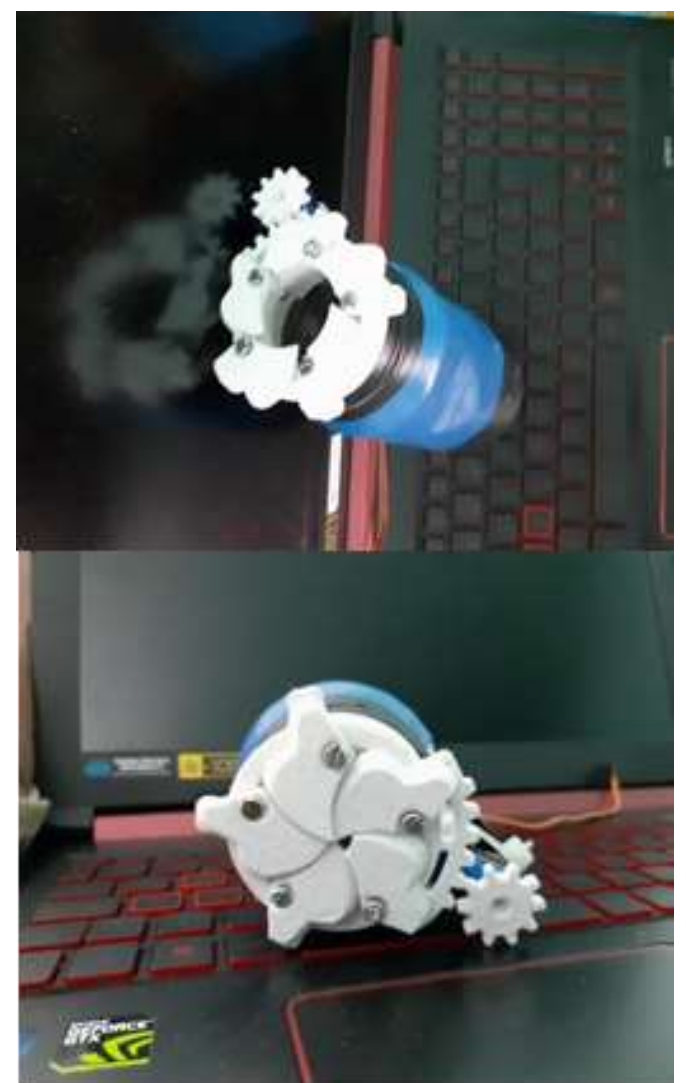

Figura 2 Contenedor de semilla utilizado

Para poder construir a nuestro DRON se utilizaron los siguientes elementos:
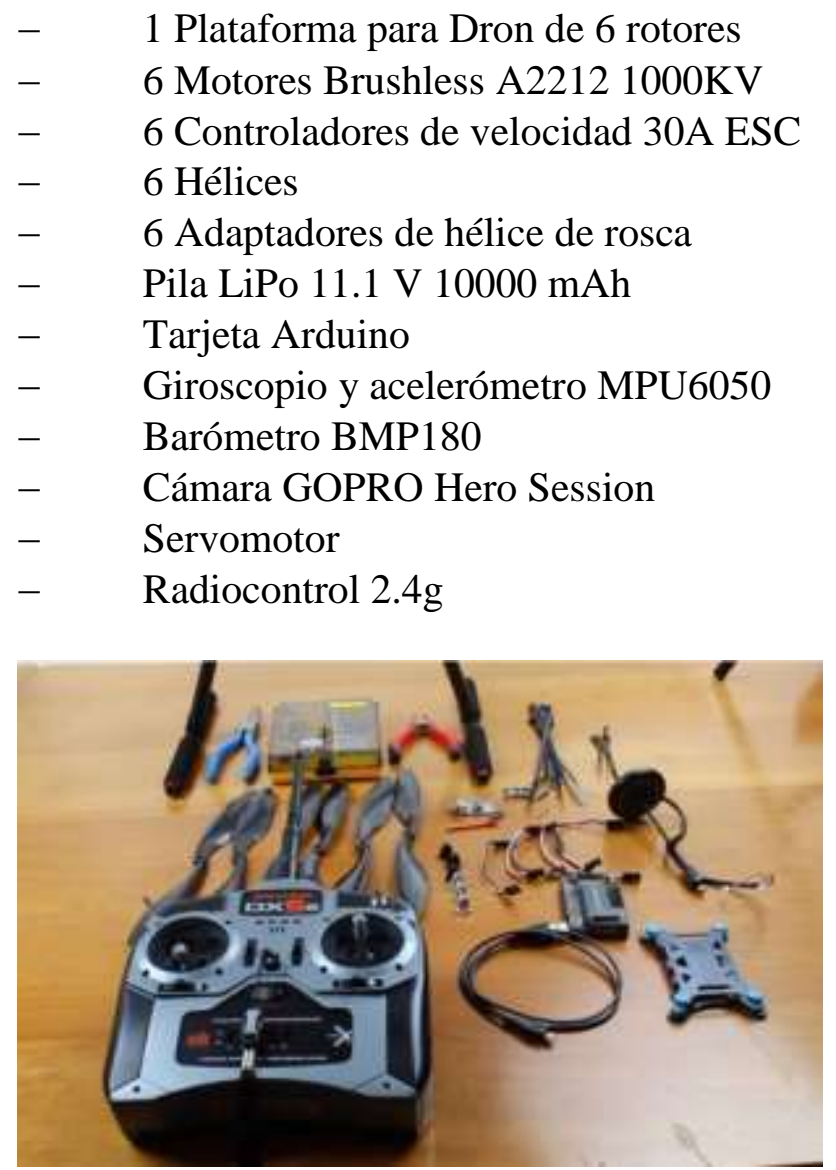

Figura 3 Elementos utilizados en el armado del dron
Se utilizó una tarjeta controladora basada en arduino para manejar al dron, y se le agregó telemetría con transmisión inalámbrica para lograr el control autónomo del mismo. La figura 4 muestra al dron ensamblado.

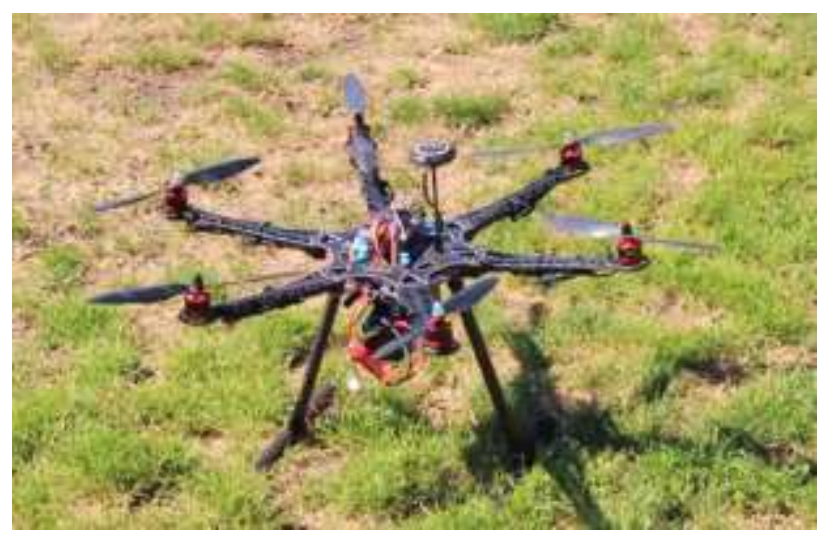

Figura 4 Dron ensamblado

A la placa controladora se le agregó un acelerómetro, un giroscopio, un barómetro y un GPS para poder tener un control más preciso sobre nuestro dron.

Una vez ensamblado al dron, se procedieron a realizar pruebas de control sobre sus motores para poder comenzar a volarlo.

Las pruebas realizadas sobre el dron en cuanto a su estabilidad se realizaron sobre una estructura metálica construida específicamente para la realización de dichas pruebas.

\section{Resultados Obtenidos}

Se realizó el modelado matemático del control de trayectorias del dron utilizando el siguiente esquema para obtener el modelo.

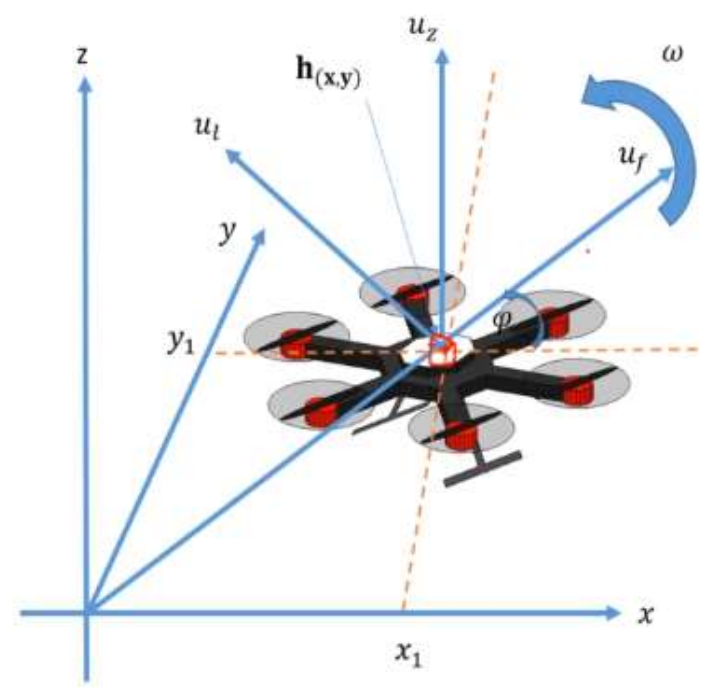

Figura 5 Esquema para modelado matemático 
Donde al dron modelado lo vamos a desplazar a lo largo de los ejes x, y y. Al dron únicamente lo vamos a rotar solo alrededor del eje $\mathrm{z}$.

El punto de control $\boldsymbol{h}_{(x, y)}$ de nuestro dron será su punto geométrico central. El dron se podrá mover de manera lateral, frontal o a lo largo del eje $z$; de ahí la nomenclatura de las velocidades $u_{l}, u_{f}, u_{z}$ y de $\omega$ como su velocidad angular.

La representación matricial se puede obtener realizando las correspondientes operaciones de rotación y traslación, por lo que se tendría la siguiente representación:

$\left[\begin{array}{c}\dot{h_{x}} \\ \dot{h_{y}} \\ \dot{h_{z}} \\ \varphi\end{array}\right]=\left[\begin{array}{cccc}\cos \varphi & -\operatorname{sen} \varphi & 0 & 0 \\ \operatorname{sen} \varphi & \cos \varphi & 0 & 0 \\ 0 & 0 & 1 & 0 \\ 0 & 0 & 0 & 1\end{array}\right]\left[\begin{array}{c}u_{f} \\ u_{l} \\ u_{z} \\ \omega\end{array}\right]$

Con el modelo matemático y utilizando el software gratuito GNU OCTAVE, se realizó la simulación del modelo para que el dron sembrador siguiera la trayectoria requerida para la dispersión de semillas sobre determinada área que se desee.

La siguiente figura muestra un ejemplo de una trayectoria que el dron sembrador seguirá y que le fue programada. En este caso es una trayectoria en línea recta rectangular para seguir los surcos de siembra en el área correspondiente.

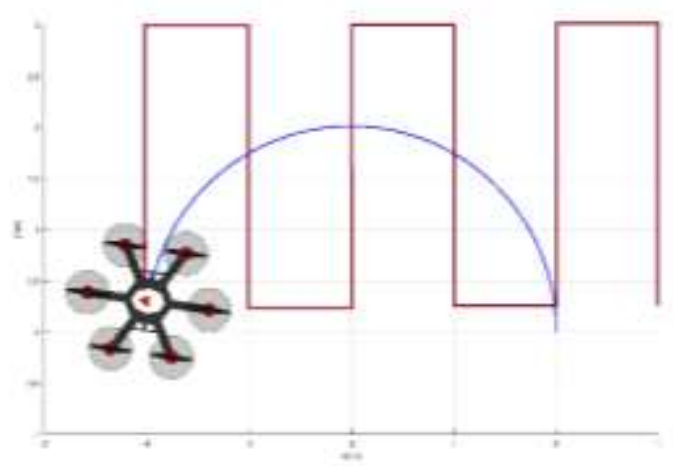

Figura 6 Ejemplo de trayectoria programada en el dron sembrador

La estabilidad del dron, la realizamos mediante la utilización de un controlador Proporcional Integral Derivativo (PID) cuya estructura sigue el siguiente esquema mostrado en la figura 7 .
Las pruebas de estabilidad realizadas mostraron que se tiene un control bastante preciso sobre la estabilidad del dron.

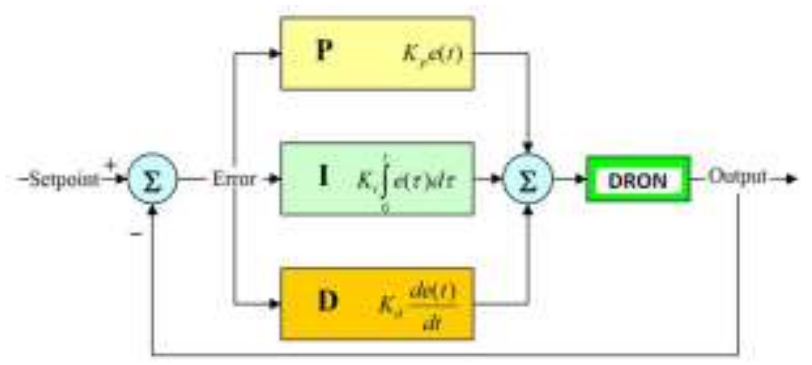

Figura 7 Estructura del PID utilizado en el dron

Para la mejora del control PID, se agregó un filtro Kalman por software al programa de control del dron.

Las pruebas en campos de cultivo de alfalfa resultaron exitosas.

La figura 8 muestra al Dron dispersando la semilla de alfalfa sobre el terreno a sembrar. Pruebas subsecuentes realizadas muestran la efectividad de nuestro dispositivo de manera autónoma.

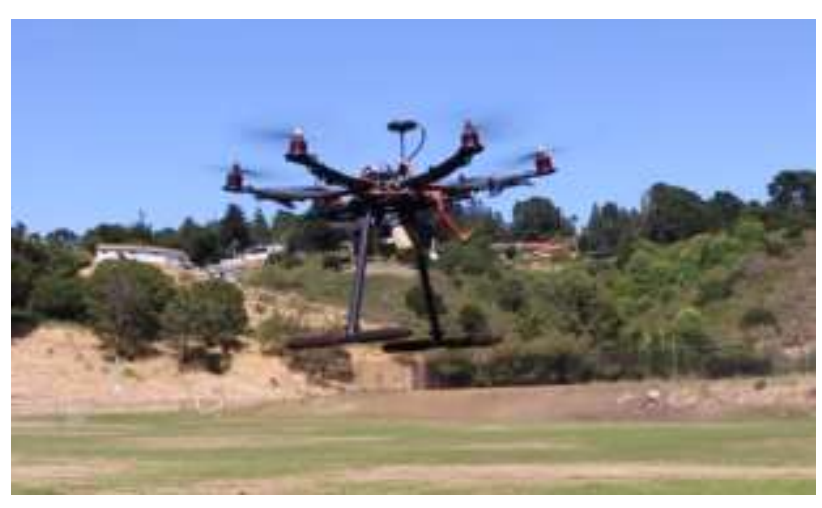

Figura 8 Prueba de dispersión de semilla de alfalfa

\section{Conclusiones}

La siembra de cultivos mediante la dispersión de semillas de forma manual es algo predominante en el sector de la agricultura.

Si se desea mejorar la producción en cualquier región y además se busca incrementar las ganancias económicas para los agricultores de determinada zona, es necesario implementar productos tecnológicos que estén al alcance de ellos. 
De igual forma, es necesario desarrollar productos innovadores que les permitan a los agricultores hacer de su jornada laboral, una jornada menos cansada, sin tanto riesgo y desgaste físico.

Para este proyecto se escogió a la alfalfa, como la especie de planta herbácea cuya semilla fue la que se utilizó para realizar las pruebas.

La densidad de siembra de la semilla de alfalfa recomendada es de 10 a $12 \mathrm{~kg}$ de semilla por hectárea, la que deberá ser depositada a una profundidad no mayor a $2 \mathrm{~cm}$. La alfalfa suele sembrarse pura, en ocasiones la siembra de alfalfa se realiza sobre terreno preparado con anticipación, pero también puede realizarse en siembra directa.

Nuestro "Dron sembrador", busca mediante la utilización de tecnología facilitar la actividad del agricultor y mediante ello hacer su labor más fructífera en todos los aspectos.

El dron construido tiene un precio de $\$ 8000.00 \mathrm{M}$. N. Consideramos que es un precio accesible ya que el costo/beneficio es muy bueno para el agricultor. En particular, considerando a la alfalfa; un corte de alfalfa está en unos $\$ 4500.00$ M. N. Es decir, en un año, la inversión del dron se paga con 2 cortes de los 6 que se realizan anualmente.

Todas las pruebas realizadas para que el dron siguiera las trayectorias modeladas en campos de cultivo para sembrar la semilla de alfalfa, arrojaron excelentes resultados.

Cabe remarcar, que si bien utilizamos a nuestro dron sembrador para la siembra de semilla de alfalfa como ejemplo, podemos utilizarlo para la siembra de semilla de cualquier cultivo.

\section{REFERENCIAS}

ENA 2017 (encuesta Nacional Agropecuaria 2017). Conociendo el campo de México. 2018. Disponible en: www.inegi.org.mx

Jerzy CHOJNACKI, Bogusława BERNER. (2018-08-30). The influence of air stream generated by drone rotors on transverse distribution pattern of sown seeds. Journal of Research and Applications in Agricultural Engineering, 63, 9-12.
Rajesh, Sing. (2017). Arduino-Based Embedded Systems, CRC Press.

Raimundo FELISMINA, Miguel SILVA, Artur MATEUS y Candida MALCA. (June 2017). Development of a Universal Seeder System to Be Applied in Drones. Journal of Advanced Agricultural Technologies, 4, 123-127.

R. Martínez Fernández. (2014). Programación en $C$. DEXTRA.

SIAP (Servicio de Información Agropecuaria y Pesquera). 2018. Disponible en: http://www.gob.mx

SIAP-SAGARPA (Servicio de Información Agroalimentaria y Pesquera-Secretaría de Agricultura, Ganadería, Desarrollo Rural, Pesca y Alimentación). 2017. Información básica, agricultura, producción anual.

Disponible en:

www.siap.sagarpa.gob.mx

William Bolton. (2016). Mecatrónica Sistemas de Control electrónico en la Ingeniería. México: Alfaomega.

VERGARA, Ángel, SALAZAR, Eduardo, ZAPATA, Oscar. (Diciembre 2017). Obtención de la función de transferencia de un motor de DC mediante el análisis de la curva de reacción. Revista de Aplicación Científica y Técnica, 3, 1-10. 